This version of the article "Knight, R., Shoveller, J., Greyson, D., Kerr, T., Gilbert, M., \& Shannon, K. (2014). Advancing population and public health ethics regarding HIV testing: a scoping review. Critical Public Health, 24(3), 283-295.” is not the final version.

We acknowledge the publisher's (Taylor \& Francis) copyright.

Here is a link to the final published version of the article: http://www.tandfonline.com/eprint/WhWyxquQKNiuqYJUntGc/full\#.Vt9yPvkrLIV 


\section{Advancing population and public health ethics regarding HIV testing: A scoping review}

Recently, scholars have called for more robust population and public health ethical frameworks to inform how the health of populations and individuals ought to be improved, including through various approaches to HIV testing practices. Our objective is to examine the breadth, range and foci of a variety of ethical issues pertaining to HIV testing approaches within the peer-reviewed literature, and how these issues address population and/or individual interests. We identify potential tensions between individual and collective approaches as well as other concerns, including equity, justice and distribution of health and risk - hallmarks of the emergent field of population and public health ethics. Based on our review, we suggest that additional theoretical work and empirical research are required in order to inform more ethically robust debates related to population HIV testing practices. Specifically problematic were consequentialist arguments that deem testing approaches as either morally permissible or impermissible without sufficiently robust empirical and/or theoretical underpinnings about how a particular approach would unfold among individuals and populations. The current review underscores the need to continue to articulate an evidence- and theoryinformed population and public health ethics pertaining to HIV testing.

Keywords: HIV testing; scoping review; population and public health ethics

\section{Introduction}

Globally, approximately 34 million people are living with HIV; and, it is estimated that nearly 30 million people have died from AIDS since the beginning of the pandemic (US Global Health Policy 2011). HIV rates vary considerably with the burden of disease concentrated among the most vulnerable and marginalized populations within and across low-, medium- and high-income countries. SubSaharan Africa accounts for approximately 67\% of global HIV infections (United Nations 2008); combined, low- and middle-income nations account for $97 \%$ of global infections (US Global Health Policy 2011). Within high-income nations, HIV rates are comparatively lower; however, vulnerable populations generally bear the burden of infection. 
In the late 1980s, the development of the HIV antibody test presented the opportunity to test individuals considered 'at risk' for HIV. During this time, calls for mandatory HIV testing of 'high risk’ groups (e.g., gay men) were advanced within various contexts (Schüklenk 1998). However, mandatory approaches were generally considered overly paternalistic and an inefficient means to curtail the spread of disease (O’Grady and Schüklenk 2009). Moreover, the lack of effective treatment, combined with concerns related to HIV stigma, led to the prioritization of informed consent over case finding (April 2010). As a result, voluntary counselling and testing (VCT) approaches to testing were generally favoured. In the VCT testing approach (often referred to as client-initiated or 'opt-in' HIV testing), the onus is on the individual to access HIV testing services and, in general, clinicians must obtain informed consent and offer pre-test counselling services before commencing blood work.

Recently, treatment and prevention technologies, including antiretroviral therapy (ART), have emerged alongside the adoption of more routine or opt-out approaches to HIV testing in many contexts, marking a departure from the approaches of the prior two decades that emphasised voluntary, opt-in testing approaches (CDC\&P 2010). First deployed within many high-prevalence antenatal settings, this approach was able to effectively reduce vertical transmission from mother to child. Routine testing approaches were soon implemented more broadly; in 2006, for example, HIV testing was "recommended for patients in all health-care settings after the patient is notified that testing will be performed unless the patient declines” (Branson et al. 2006) by the United States Centre for Disease Control and Prevention. In 2007, the World Health Organization recommended that all adult patients in high-prevalence settings be tested unless they explicitly 'opt out'.

Indeed, there is a strong public health impetus to decrease the spread of HIV by identifying those who are infected with the aim of providing treatment and preventing onward transmission (e.g., through behavioural counselling). The provision of HIV testing has been considered as a means to benefit individuals, as well as a mechanism to improve population health by reducing HIV incidence and prevalence. As a result, there has been a proliferation of efforts to test both individuals 
and populations considered 'at risk' for HIV acquisition. Recently, the emergence of empirical evidence supporting the role of ART and 'treatment as prevention' (in which viral loads are suppressed, thereby preventing onward transmission) (Montaner et al. 2010) has further altered the landscape within HIV testing. The capacity for ‘treatment as prevention’ to reduce HIV prevalence and incidence at a population level (beyond the known individual-level benefits of ART) catalyzed the seek, test and treat approach, and more recently the cascade/continuum of care approach, whereby access to testing is posited as the first step in a pathway leading to suppressed viral loads and reduction of HIV incidence (Cohen et al. 2011).

\section{Ethics and HIV testing}

As approaches to HIV testing have shifted over time, so have the ethical debates surrounding and informing these practices. Generally, these perspectives have been informed by either deontological or utilitarian ethical frameworks. Utilitarian approaches tend to draw on the work of John Stuart Mill to delineate the circumstances in which intervention on an individual (the patient) by a collective (the population or State) is acceptable - namely "that the only purpose for which power can be rightfully exercised over any member of a civilized community, against his will, is to prevent harm to others” (Mill 1860 p.9). Deontological approaches generally focus on principles that emphasize an individual's right to autonomy and consent, as well as the duty to minimize harm to others.

Over the past decade, a number of theorists have called for a more robust population and public health ethics that expands upon traditional orientations of bioethics that focus primarily on the interests of individuals, often in clinical encounters. For example, Bayer and Fairchild (2004) describe how, within several instances of infectious disease outbreaks (e.g., the SARS outbreak in 2002), traditional bioethical frameworks were deemed to be insufficient in providing a moral evaluation of various public health actions thought to be required in order to effectively (and morally) suppress the spread of disease (e.g., quarantining asymptomatic individuals thought to be exposed in the absence of a diagnostic test). 
Thus, in part, population and public health ethics emerged out of a series of controversies between the central commitments of public health (e.g., with an emphasis on collective well-being) and bioethics (e.g., with an emphasis on individual-level concerns) (Bayer and Fairchild 2004).

As the nascent field of population and public health ethics developed it also took up ethical issues related to how health is distributed (inequitably) within and across populations (Daniels 2006). As a result, population and public health ethics seeks to provide a critical evaluation of questions concerning possible, actual and proposed public health measures in order to more fully consider and negotiate a 'balance' of individual and collective interests and health outcomes (Coggon 2012). As Daniels (2006) points out, an inherent challenge within this field is the need to incorporate a variety of theoretical and interdisciplinary approaches in order to situate both the health of a population and individuals within a framework that emphasizes social justice and health equity (Daniels 2006).

The current paper provides a critical evaluation of the peer-reviewed literature to identify how ethical issues have been addressed since 2001 - recognizing the need to advance population and public health ethics in this area in light of new HIV testing and treatment strategies, as well as evolving testing approaches. We describe how issues related to the changing context of HIV are connected to practices that target/prioritise testing efforts. We also examine the influences of HIV stigma, as well as the structural and agentic factors that influence HIV-related experiences. Finally, we identify knowledge gaps and areas that require additional theoretical or empirical advancements in order to advance population and public health ethical considerations within the realm of HIV testing.

\section{Methods}

We drew on scoping review methods outlined by Arksey and O'Malley's (2005) in order to acquire a set of peer-reviewed articles discussing the ethics of various HIV testing approaches that we could critically examine through a population and public health ethics perspective. Our aim is to systematically interrogate the ongoing debate and discussion, and describe how this has evolved as the context of HIV also has 
changed (e.g., treatment opportunities and new knowledge about transmission). As we conducted our review, we sought to answer the following research questions: How have the interests of individuals and populations been considered with respect to what HIV testing approaches ought to be taken? Specifically, how does the literature address: HIV-related stigma; agentic and structural factors of HIV infection; and the targeting or prioritising of specific populations or population sub-groups? And, finally, what are the strengths, weaknesses and areas that require more attention?

\section{Search and inclusion/exclusion strategy}

We searched the MEDLINE database (via the OvidSP interface) for articles on HIV testing and ethics by using subject headings for: HIV and “mass screening”, HIV infection diagnosis, and AIDS serodiagnosis; and subject headings, subheadings and keywords for ethics. We limited the scope of the search to English-language articles about humans that were published from 2001-2012, resulting in a total of 155 articles. In order to be eligible for inclusion, articles had to include normative ethical discussions about the moral justifiability of approaches to HIV testing. Studies that only described a group's beliefs or preferences about ethical issues were excluded. Upon completion of article-level screening for inclusion, reference lists of included articles were scanned for additional relevant articles (citation snowballing), which resulted in 4 additional articles for inclusion. Finally, one article from a co-author's personal library collection was included, resulting in a total of 35 articles included in the review (see Table 1).

\section{Analysis}

Each article was reviewed to identify instances in which ethical implications of HIV testing approaches were discussed; the extracted examples were summarized in a Microsoft Excel spreadsheet. Employing thematic analysis techniques, we used a combination of inductive and deductive approaches to identify patterns across the articles and to develop an overall interpretation (Fereday \& Muir-Cochrane 2006) of the literature from a population and public health ethics perspective. First, in order to understand how the literature has dealt with considerations of population and public 
health ethics and HIV testing, we identified three categories that reflect important influences on HIV infection and experiences: (1) HIV-related stigma; (2) prioritising, targeting and seeking testing efforts; and (3) structural and agentic factors pertaining to HIV infection and testing experiences. We drew on the ways in which these three issues have been identified in previous literature to deduce and articulate their conceptual connections with population and public health ethics and HIV testing. We also inductively derived new ideas and interpretations from our analysis of the extracted data in order to further inform our critical interpretation of the overall thematics represented in our sample.

\section{Findings}

\section{HIV testing and HIV-related stigma}

Since the beginning of the HIV epidemic, HIV-related stigma has been positioned as a moral dimension of various approaches to HIV testing. For example, there has been a consistent concern that a move towards mandatory approaches could drive the epidemic 'underground' because those who are considered most 'at-risk' for HIV acquisition are those individuals already highly vulnerable to stigmatization (e.g., gay/bisexual/MSM men, drug users and sex workers) (Bayer and Fairchild 2006). Smith et al. (2011) argue that opt-out frameworks will be especially stigmatising for population sub-groups that are already at increased risk of experiencing hardships as a result of their social position. For example, stigma associated with a positive diagnosis has been described as having particularly harmful effects on women, with potential consequences including abandonment by their partners and community (Kippax 2006). However, as Csete and Elliott (2006) acknowledge, there is a need for more empirical research to investigate the stigmarelated effects of a positive diagnosis (e.g., rates of depression, suicide, abandonment, violence and other abuses).

As ART became more widely available, the 'opt-in' approach to testing also has been criticised as being overly 'exceptionalistic' - that is, it was argued that HIV was being treated differently in comparison with other communicable and sexually transmitted infections (Bayer and Fairchild 2006). In 2002, De Cock et al., suggested 
that 'exceptionalist' practices may exacerbate HIV stigma rather than preventing it. In this argument, it is instead suggested that routine approaches will reduce stigma because everyone is considered as a target for testing; as a result, specific population sub-groups will no longer be targeted as the 'risky other'. In general, these arguments rely on the assumptions that individual-level concerns (e.g., an emphasis on a fully informed and voluntary process) may no longer need to be prioritized in light of treatment advances (e.g., ART decreases likelihood of onward transmission and is now more widely available within many global contexts) and social change (e.g., laws that prohibit discrimination based on one’s HIV status) (Gostin 2006). Other authors remain less convinced, arguing that opt-out approaches do not directly address the underlying issues associated with stigma (Meera and Sreeram 2007), nor do they adequately take into account the reality that HIV-related stigma is alive and well, even in the most 'progressive' societies (Fields and Kaplan 2011). Relatedly, Kippax (2006) argues that, without understanding or appreciating the cultural and social conditions under which individuals are being tested, opt-out approaches may inadvertently increase stigma and discrimination (although Kippax does not describe the pathways through which this effect is likely to be manifested).

Thus, two dominant themes emerged with respect to explaining how HIV testing practices are perceived to be interconnected with HIV-related stigma. First, there is a collection of arguments that position HIV stigma as accumulating through routine testing approaches. Within this way of thinking, increasing the number of HIV positive people tested through routine testing would imply that more HIV positive people will become aware of their status and therefore exposed to HIV-related stigma. Conversely, another set of arguments exists whereby making HIV testing more routine and normalised, HIV stigma will be reduced. However, both of these streams of arguments related to stigma suffer from empirical and theoretical limitations. Specifically, from a conceptual perspective, stigma is frequently positioned as a binary outcome (e.g., either an individual experiences stigma or does not). In developing a more robust population and public health ethics related to HIV testing, we argue that HIV-related stigma be conceived of as something more than an abstract phenomenon that 'happens' to an individual in order to open up new theoretical 
spaces to conceptualise HIV-related stigma as a question of systemic social and structural inequalities related to the intersections of power, culture and difference (Parker and Aggleton 2003). Such a conceptualisation of HIV-related stigma emphasizes the relational dimensions of the health and social effects of stigma on health, providing opportunities to highlight the social processes that serve to differentially (re)produce social and structural inequalities (e.g., related to: class; gender; race; socio-economic status; culture) within and across global and local contexts (Scambler 2009). Without conceptualising stigma in more sophisticated ways, the moral dimensions of arguments pertaining to the links between HIV stigma and HIV testing approaches will remain predominantly individualistic, hampering abilities to sufficiently address issues of equity at a population level.

Several of the authors (e.g., Csete and Elliott 2006; Kippax 2006) also suggest more empirical evidence is needed to determine how various testing approaches (e.g., opt-out versus opt-in) interact with HIV-related stigma. In part, the lack of empirical population-level data related to the social and health effects of HIV-related stigma and HIV testing approaches stems from relatively limited methodological tools (Parker and Aggleton 2003). Nonetheless, innovative research methods continue to emerge within this field; for example, a recent study by Jurgensen et al. (2013) used a pairmatched randomized cluster trial to examine differences in stigma over time among those accessing voluntary testing and counselling versus home-based testing. While we acknowledge that new evidence in this field needs to be considered critically and contextually, we suggest that more evidence should be developed to inform ethical debates in this field - particularly within consequentialist arguments that rely on potential outcomes to delineate morally justifiable actions.

\section{Prioritising, targeting and seeking: Addressing individual- and population-level} interests

Within our review, the prioritisation and targeting of HIV testing to specific population subgroups was discussed in two ways: (1) with respect to the ethics of mandatory testing approaches with health care practitioners, pregnant women and within correctional institutions; (2) with respect to seek, test and treat approaches. 
Health care practitioners were described within two articles (Leung 2001; Aziz 2007) as being a group that may require special consideration related to mandatory HIV testing practices, given that they may engage in medical procedures that elevate the risk of HIV acquisition/transmission. Leung (2001) identifies the deployment of limited resources as an important consideration, acknowledging that while nurses have the duty not to impose risks to their patients, suggesting that they ought to know their status, mandatory testing of nurses cannot be justified, given that the high number of nurses that must be tested in order to prevent one case of nurse-to-patient infection would require too large a resource investment (particularly of concern for low-resource settings). Basu et al. (2005) suggest that, within the context of correctional institutions, mandatory testing may only be suitable within longer-stay prisons, where there would be more opportunities for sufficient counselling and treatment in the event of a positive diagnosis. Nonetheless, as O’Grady and Schüklenk (2009) point out, there is a dearth of ethical analyses surrounding mandatory HIV testing approaches and future debates and contributions are likely needed in this area.

Pregnant women are a special case within the literature in this area. In general, it was argued that, as perinatal HIV transmission can be reduced through the use of timely treatment, opt-out approaches are warranted when women access gynaecologic/obstetric care. Fields and Kaplan (2011) argue, however, that while there can be significant benefits to both mother and fetus from HIV testing, opt-out approaches may involve a higher degree of coercion for pregnant women (e.g., through subtle/overt pressure from partners, family and clinicians). Others argue that gendered health-seeking patterns are more likely to expose women and girls to health care service provision and, conversely, exclude men and boys - thereby eliminating the 'universality' of opt-out practices and placing a tacit burden of testing on women (Rennie and Behets 2006; April 2010).

Most argued that, while it is noble to improve pregnancy outcomes, including the HIV status of foetuses and neonates, it is unethical to construct the rights of the foetus as separate from (or opposed to) the rights of the woman. Salang Seloilwe (2011) accepts that targeting pregnant women may violate arguments regarding bodily sovereignty, but argues that this concern is outweighed by the principle of justice, 
whereby the distribution of benefits and burdens is particularly concerned with those who cannot protect their own interests (e.g., the foetus). Conversely, Armstrong (2008) argues that mandatory testing may represent a denial of dignity to pregnant women, yet suggests that if mandatory testing for pregnant women is not justifiable in high prevalence settings, then it cannot ever be morally justifiable. Ultimately, these discussions reveal how each of these testing approaches tends to rely on the medicalization of women's bodies. Indeed, while many testing approaches aim to be 'universal', it is clear that women - particularly pregnant women - represent a group that is subject to enhanced HIV surveillance, thereby (re)contributing to a 'hyperfeminisation' of HIV testing.

Only two papers in our review discussed the ethics of seek, test and treat approaches. Vonn (2012) worries that, in practice, 'seeking' strategies may rely on coercive or incentivizing techniques among economically disadvantaged populations and potentially disregard the "criminal jeopardy" that individuals will be exposed to should they receive a positive diagnosis (e.g., in Canada, it is illegal to withhold one's HIV status with sex partners) - processes and impacts that have yet to be demonstrated through rigorous empirical investigation. Bayer (2010), on the other hand, argues that ethical analyses that focus exclusively on individual-level concerns are not useful when considering 'seeking' strategies, given the severity of the HIV pandemic. Instead, Bayer (2010) calls for analyses that emphasize both collective and individual well-being to determine whether or not various seeking approaches can be justified to increase testing rates.

From a consequentialist approach (i.e., the view that the right act is determined by its capacity to promote the greatest amount of good and render the least amount of harm), Dawson (2011) describes how opt-out testing practices are a population health intervention that benefits future populations and are therefore morally justifiable. O’Grady and Schüklenk (2009) argue that, whereas previously mandatory approaches had little benefit to offer individuals, given the advances in ART, early detection is now critical and therefore opt-out approaches to HIV testing are morally justifiable. As a result, mandatory testing has increasingly been positioned within the literature as both a means to help the individual to gain opportunities to 
improve health, as well as an opportunity to prevent onward transmission and future harm to others. However, April (2010) argues that any justification for opt-out testing must rely on the individual-level benefits, not the population, due to a "lack of evidence” supporting the often promoted population-level benefits. This and other examples do not appear to acknowledge the empirical evidence indicating favorable population-level health benefits (e.g., Montaner et al. 2010), which we find to be surprising given the high profile of seek, test and treat within contemporary HIV discourses and practices. For example, Vonn’s (2012) ethical assessment of seek, test and treat relies solely on policy documents, excluding empirical assessments of health outcomes. While this may reflect a publication 'lag', we also suggest this is a 'high priority' area within the field of population and public health ethics and hope that future debates in these areas will be more informed by available and forthcoming evidence. $^{1}$

In general, we were struck by the lack of discussions of ethical issues related to targeting or prioritising HIV testing programs. Targeting population sub-groups is a hallmark within infectious epidemiology and relates to the distribution of limited resources. Sexual identity and other social characteristics (e.g., ethnicity; social class) are widely used as proxies to target HIV interventions, in part because they are thought to be efficient ways of targeting HIV interventions (including testing programmes), especially to vulnerable, so-called 'high-risk' groups. For example, some HIV testing strategies specifically target testing messages at men who have sex with men and African-origin immigrants (e.g., National AIDS Trust 2012), and ethical issues associated with these practices were absent within articles in the current review. However, outside of the current review and within the population health literature more broadly, there is emerging concern that targeted public health intervention approaches in general (e.g., those based solely or primarily on social characteristics) may exacerbate health inequity (Frohlich and Potvin 2008). A recent paper, for example, suggests that intervening approaches that focus on discrete social

\footnotetext{
${ }^{1}$ For a recent example (outside of the field of HIV testing) of a critical interrogation of the population and public health ethical considerations related to available individualand population-level evidence, see Thompson's (2013) discussion of an HPV vaccination program in a Canadian-based school.
} 
differences to address health may (unintentionally) result in positive discrimination or stigmatization (McLaren, McIntyre, and Kirkpatrick 2009). Targeted interventions also have been critiqued for burdening specific population subgroups with an intervention, while aiming to benefit those not explicitly targeted through a dispersion of benefits (e.g., reducing disease in a population will reduce the likelihood of disease acquisition among those not targeted) (O’Neill 2011). Unfortunately, the literature related to the ethics of HIV testing has yet to unpack the ethical implications of targeted interventions and we recommend these as areas of future examination.

\section{Structural and agentic factors related to HIV infection and testing experiences}

HIV infection and testing experiences are linked to both agentic practices (e.g., capacity to deploy a range of causal powers with respect to HIV testing) as well as to structural features within and across populations (e.g., socio-economic inequalities). Within our review, we found that the ethical considerations related to structural and agentic influences were frequently implicated in discussions related to paternalism and opt-out testing approaches. Generally, we found the discussions related to structure, agency and HIV testing tended to emphasize individualistic concerns by positioning the unit of analysis in an HIV test as an individual. In this light, the capacity for HIV testing to operate as a preventative tool was generally conceptualised through counselling individual behaviour change. For example, DeCock et al (2002) criticise the limited ability of VCT to function as a preventative tool in high-prevalence settings due to the prioritization of individual-level considerations of informed consent, counselling and anonymity. Csete and Elliott (2006) argue that, by eliminating (or reducing) the use of pre-test counselling, opt-out HIV testing loses its power as a prevention tool. We suggest that we may require a conceptual 'shift' within these moral discussions that position population-level HIV testing practices as both a societal resource (structure), as well as a mechanism that influences the nexus of individual agency (e.g., the ability to deploy a range of causal powers related to sexual health practices) and the (re)production of hierarchal social relations (e.g., norms in which people can engage to promote more socially just health and social outcomes). While we agree with others who have suggested that scaling up 
individually based interventions does not, in itself, represent a structural intervention (McLaren, McIntyre and Kirkpatrick 2010), we argue that the various HIV testing approaches can be more meaningfully considered as interventions that have the capacity to be preventative at an individual level (e.g., through pre- and post-test counseling) and structural level. For example, Knight et al (2012) describe how sexual health clinical encounters (including HIV testing) can provide opportunities for young men to engage in social interactions that can begin to deconstruct social structures that (re)produce social injustices (e.g., heteronormativity; hegemonic masculinity; misogyny; HIV-related stigma). While some articles in the current review allude to HIV testing as having a transformative capacity at a structural level (e.g., within arguments suggesting HIV-related stigma can be reduced within opt-out approaches), these arguments neglected to identify the specific pathways in which more equitable outcomes can be advanced.

\section{Conclusion}

The issues raised within the current review offer a glimpse into the moral complexities associated with HIV testing approaches. Here, we described how the trend from opt-in to opt-out approaches is being morally justified or critiqued and shown how various testing approaches intersect with HIV-related stigma, targeted approaches to testing and structure and agency. Our review does not seek to provide support for or against a specific testing approach. We also acknowledge that many ethical debates related to HIV testing practices take place outside of academic writing/journals (e.g., in the public sphere). However, our review of the current state of normative debates in the peer-reviewed literature related to population and public health ethics and HIV testing offers a distillation of where efforts are being focused, and suggests areas where new efforts could yield potential gains.

Based on our review of this literature, we suggest that additional theoretical work and empirical research are required in order to inform more ethically robust debates related to HIV testing practices. Specifically problematic are consequentialist arguments that deem testing approaches as either morally permissible or impermissible without sufficiently robust empirical and/or theoretical underpinnings about how a particular 
approach would unfold among individuals and populations. A robust population and public health ethics must address issues related to the intersections of individual-level factors (e.g., consent; autonomy), as well as the structural and social inequalities that concentrate illness within the most vulnerable populations. While we agree with others who have cautioned that over-focusing on 'the empirical' within 'the normative' risks losing sight of the analytic method required in moral evaluation (Goldenberg 2005), we also argue that the apparent lack of theorising and empiricising deployed in descriptions of HIV-related stigma and structural inequalities reveals the need for a renewed emphasis on interdisciplinary approaches across the humanities (e.g., philosophy; history; law), social sciences (e.g., sociology; political sciences; economics) and health sciences (e.g., medicine; epidemiology).

We also are critical of the arguments that assume a uniformity of agency within and across populations, as these arguments tend to be fundamentally threatened by the reliance on an assumption that each individual has an equal degree of agency and can volunteer for testing as needed (and moreover has the knowledge and motivation to do so). Indeed, from within the realm of public health, this approach relies exclusively on individual-based approaches to give knowledge to the individual in order that they access testing (and therefore disregards social structures and the distribution of agentic privilege). In this capacity, these arguments fail to attend to the philosophical underpinnings of population and public health ethics that emphasizes equity, justice and considerations of structural distributions of health and illness.

Philosophical and ethical deliberations are much-needed processes when negotiating a balance of the interests of individuals and populations, particularly in the quickly evolving context of HIV. For example, new HIV-related technologies and evidence continuously emerges in this rapidly changing area, including home-based tests, online testing programs, as well as new pre- and post- exposure prophylaxis (within some settings), bringing forward new challenges with respect to their implications. Whether we can or should attain a state of global consensus or equilibrium about the moral underpinnings of public health actions related to HIV intervention and prevention remains unclear; however, the current review underscores 
the need to continue to articulate and develop a population and public health ethics within this substantive area.

\section{References}

April, M. D. 2010. Rethinking HIV exceptionalism: the ethics of opt-out HIV testing in sub-Saharan Africa. Bulletin-World Health Organization 88(9): 703-708.

Arksey, H., and O'Malley, L. 2005. Scoping studies: towards a methodological framework. International Journal of Social Research Methodology 8(1): 19-32.

Armstrong, R. 2008. Mandatory HIV testing in pregnancy: is there ever a time? Developing World Bioethics 8(1): 1-10.

Aziz, B. 2007. Testing times. Nursing management 14(6) 8-9.

Basu, S., Smith-Rohrberg, D., Hanck, S., and Altice, F. L. 2005. HIV testing in correctional institutions: evaluating existing strategies, setting new standards. AIDS and Public Policy Journal 20(1-2): 3-24.

Bayer, R., and Fairchild, A. L. 2004. The Genesis of Public Health Ethics. Bioethics 18(6): 473-492.

Bayer, R., and Fairchild, A. L. 2006. Changing the paradigm for HIV testing-the end of exceptionalism. New England Journal of Medicine 355(7): 647-649.

Bayer, R. 2010. Mass Testing and Mass Treatment for Epidemic HIV: The Ethics of Medical Research is No Guide. Public Health Ethics 3(3): 301-3.

Bennett, R. 2007. Routine antenatal HIV testing and informed consent: an unworkable marriage? Journal of Medical Ethics 33(8): 446-448.

Branson, B. M., Handsfield, H. H., Lampe, M. A., Janssen, R. S., Taylor, A. W., Lyss, S. B., and Clark, J. E. 2006. Revised recommendations for HIV testing of adults, adolescents, and pregnant women in health-care settings. Morbidity and Mortality Weekly Report 55(RR-14): 1-17.

Brewster, D. 2011. Science and ethics of human immunodeficiency virus/acquired immunodeficiency syndrome controversies in Africa. Journal of Paediatrics and Child Health, Ethics of HIV in Africa 47(9): 646-655.

Cameron, T. 2002. Mandatory HIV testing of newborns in New York State. Journal of Health and Social Policy 14(3): 59-78. 
Celada, M. T., Merchant, R. C., Waxman, M. J., and Sherwin, A. M. 2011. An ethical evaluation of the 2006 Centers for Disease Control and Prevention recommendations for HIV testing in health care settings. American Journal of Bioethics 11(4): 31-40.

Centers for Disease Control and Prevention. 2010. Expanded HIV Testing and Trends in Diagnoses of HIV Infection—District of Columbia, 2004-2008. Journal of the American Medical Association 304(3): 264-266.

Coggon, John. 2012. What Makes Health Public? A critical evaluation of moral, legal and political claims in public health. Cambridge: Cambridge University Press.

Cohen, S. M., Van Handel, M. M., Branson, B. M., Hall, I. H., Hu, X., and Koenig, L. J. 2011. Vital signs: HIV prevention through care and treatment-United States. MMWR Morb Mortal Wkly Rep 60(47): 1618-1623.

Csete, J., and Elliott, R. 2006. Scaling up HIV testing: human rights and hidden costs. HIV/AIDS Policy and Law Review 11(1): 5-10.

Daniels, N. 2006. Equity and Population Health: Toward a Broader Bioethics Agenda. Hastings Center Report 36(4): 22-35.

Dawson, A. 2011. Public health ethics and the justification of HIV screening. American Journal of Bioethics 11(4): 48-49.

De Cock, K. M., Mbori-Ngacha, D., and Marum, E. 2002. Shadow on the continent: public health and HIV/AIDS in Africa in the 21st century. The Lancet 360(9326): 67-72.

Dixon Mueller, R. 2007. The sexual ethics of HIV testing and the rights and responsibilities of partners. Studies in Family Planning 38(4): 284-296.

Fereday, J., and Muir-Cochrane, E. (2006). Demonstrating Rigor Using Thematic Analysis: A Hybrid Approach of Inductive and Deductive Coding and Theme Development. International Journal of Qualitative Methods 5(1): 80-92.

Fields, L., and Kaplan, C. 2011. Opt-out HIV testing: An ethical analysis of women's reproductive rights. Nursing Ethics 18(5): 734-742.

Frohlich, K. L., and Potvin, L. 2008. Transcending the known in public health practice: The inequality paradox: The population approach and vulnerable populations. American Journal of Public Health 98(2): 216-221.

Goldenberg, M. J. 2005. Evidence-based ethics? On evidence-based practice and the "empirical turn" from normative bioethics. BMC Medical Ethics 6(1): 11. 
Gostin, L. O. 2006. HIV screening in health care settings. JAMA: the Journal of the American Medical Association 296(16): 2023-2025.

Johansson, K.A., Pedersen, K.B. and Andersson, A. K. 2011. HIV testing of pregnant women: An ethical analysis. Developing World Bioethics 11(3): 109-119.

Jurgensen, M., Sandoy, I.F., Michelo, C. and Fylkesnes, K. 2013. Effects of home-based Voluntary Testing and Counselling on HIV-related stigma: findings from a clusterrandomized trial in Zambia. Social Science and Medicine.

Knight, R., Shoveller, J., Oliffe, J., Gilbert, M., \& Goldenberg, S. 2012.

Heteronormativity Hurts Everyone: Experiences of young men and clinicians with STI/HIV testing in British Columbia, Canada. Health: An Interdisciplinary Journal for the Social Study of Health, Illness and Medicine.

Kippax, S. 2006. A public health dilemma: A testing question. AIDS Care 18(3): 230235.

Leung, W. C. 2001. Should screening of student and qualified nurses for bloodborne infections be compulsory and infected individuals excluded from work? Nursing Ethics 8(2): 133-141.

Macklin, R. 2005. Scaling up HIV testing: ethical issues. Health and Human Rights 8(2): 27-30.

McLaren, L., McIntyre, L., \& Kirkpatrick, S. 2009. Rose’s population strategy of prevention need not increase social inequalities in health. International Journal of Epidemiology 39(2): 372-377.

McQuoid-Mason, D. 2007. Routine testing for HIV-ethical and legal implications. South African Medical Journal 97(6): 416.

Meera, R., and Sreeram, S. 2007. Provider-initiated testing and counselling in India for HIV. Indian Journal of Medical Ethics 4(4): 170.

Mill, John Stuart. 1860. On Liberty. John Stuart Mill's 5 Legendary Lectures on Personal Liberty. In Harvard Classics, 25. 2010.

Montaner, J.S., Lima, V., Barrios, R., Yip, B., Wood, E., Kerr, T., Shannon, K., Harrigan, R., Hogg, R., Daly, P. and Kendall, P. 2010. Association of highly active antiretroviral therapy coverage, population viral load, and yearly new HIV diagnoses in British Columbia, Canada: a population-based study. The Lancet 376(9740): 532539.

National AIDS Trust. 2012. HIV Testing Action Plan - to reduce late HIV diagnosis in the UK. United Kingdom. 
Ngwena, C. G., and Cook, R. J. 2008. HIV/AIDS, pregnancy and reproductive autonomy: Rights and duties. Developing World Bioethics 8(1): iii-vi.

O'Grady, B., and Schüklenk, U. 2009. Rethinking mandatory HIV testing. Bioethics 23(8): ii-ii.

O’Neill, O. 2011. Broadening bioethics: Clinical ethics, public health and global health. Presented at Nuffield Council's Annual Lecture at the Royal Society of Arts.

Parker, R., and Aggleton, P. 2003. HIV and AIDS-related stigma and discrimination: a conceptual framework and implications for action. Social Science \& Medicine 57(1): 13-24.

Pierce, M. W., Maman, S., Groves, A. K., King, E. J., and Wyckoff, S. C. 2011. Testing public health ethics: Why the CDC's HIV screening recommendations may violate the least infringement principle. Journal of Law, Medicine and Ethics 39(2): 263271.

Powderly, K. 2001. Ethical and legal issues in perinatal HIV. Clinical Obstetrics and Gynecology 44(2): 300.

Rennie, S., and Behets, F. 2006. Desperately seeking targets: the ethics of routine HIV testing in low-income countries. Bulletin-World Health Organization 84(1): 52-57.

Rennie, S., and Mupenda, B. 2008. Ethics of mandatory premarital HIV testing in Africa: The case of Goma, Democratic Republic of Congo. Developing World Bioethics 8(2): 126-137.

Salang Seloilwe, E. 2011. Commentary on 'Opt-out HIV testing: An ethical analysis of women's reproductive rights'. Nursing Ethics 18(5): 743-745.

Scambler, G. 2009. Health-related stigma. Sociology of Health \& Illness 31(3): 441-455.

Schüklenk, U. 2003. AIDS: Bioethics and public policy. New Review of Bioethics 1(1): 127-144.

Smith, W. 2011. When informed consent meets the everyday. American Journal of Bioethics 11(4): 43-45.

United Nations. 2008. 2008 report on the global AIDS epidemic. Geneva: Joint United Nations Programme on HIV/AIDS. Available from: http://data.unaids.org/pub/ GlobalReport/2008/JC1510_2008GlobalReport_en.zip. 
United States Global Global Health Policy. 2011. The Global HIV/AIDS Epidemic, November, 2011. Accessed on April 15, 2012 at:

http://www.kff.org/hivaids/upload/3030-16.pdf.

Vernillo, A. 2011. Routine opt-out HIV testing in dental health care-Its implementation and the advancement of public health. American Journal of Bioethics 11(4): 46-48.

Vonn, M. 2012. British Columbia’s “seek and treat” strategy: a cautionary tale on privacy rights and informed consent for HIV testing. HIV/AIDS Policy \& Law Review 16: 15-18.

Wahlert, L., and Fiester, A. 2011. The re-queering of HIV testing practices and the reinforcement of stigma. American Journal of Bioethics 11(4): 41-43.

Wynia, M. K. 2006. Routine screening: Informed consent, stigma and the waning of HIV exceptionalism. American Journal of Bioethics 6(4): 5-8.

\section{Acknowledgements}

Lead author Knight is supported by a doctoral Canada Graduate Scholarship funded by the Canadian Institutes of Health Research and the Canadian Association for HIV Research and he holds a Bridge Fellowship from the Bridge Program. 
Table 1. List of articles reviewed by publication, primary author and approach to HIV screening

\begin{tabular}{ll} 
Pub Year & Authors, Primary \\
\hline 2010 & April \\
2008 & Armstrong \\
2007 & Aziz \\
2005 & Basu et al. \\
2010 & Bayer \\
2006 & Bayer et al. \\
2007 & Bennett \\
2011 & Brewster \\
2002 & Cameron \\
2011 & Celada et al. \\
2006 & Csete et al. \\
2011 & Dawson \\
2002 & De Cock \\
2007 & Dixon-Mueller \\
2011 & Fields et al. \\
2006 & Gostin \\
2007 & Hanssens \\
2011 & Johansson et al. \\
2006 & Kippax \\
2001 & Leung \\
2005 & Macklin \\
2007 & McQuoid-Mason \\
2007 & Meera et al. \\
2008 & Ngwena et al. \\
2009 & O'Grady et al. \\
2011 & Pierce et al. \\
2001 & Powderly \\
2006 & Rennie et al. \\
2008 & Rennie et al. \\
2011 & Salang Seloilwe \\
2011 & Smith \\
2011 & Vernillo \\
2012 & Vonn \\
2011 & Wahlert et al. \\
2006 & Wynia \\
&
\end{tabular}

